\title{
Comunicación
}

\section{Estudio retrospectivo de patologías músculo esqueléticas de perros en un Hospital Veterinario en Chile, entre los años 2014 y 2017}

\author{
Retrospective study of musculoeskeletal pathologies in dogs at a Veterinary \\ Hospital in Chile, from 2014 to 2017
}

\author{
Christof Fischer Wiethuchter ${ }^{1,2}$, Cristina Bailén Bolaño ${ }^{1}$
}

\section{Resumen}

Se realizó un estudio retrospectivo de patologías músculo esqueléticas en perros en el Centro Veterinario Alemán Kleintierklinik de la Ciudad de San Pedro de la Paz, Chile, en el periodo comprendido entre septiembre del año 2014 a junio del año 2017. Del total de las 2000 fichas clínicas analizadas, 144 fichas presentaban pacientes con algún problema en el sistema músculo esquelético. Las patologías músculo esqueléticas con mayor incidencia fueron las fracturas $(40,27.8 \%)$, luxación patelar $(31,21.5 \%)$, ruptura del ligamento cruzado craneal $(25,17.4 \%)$ y displasia de cadera $(23,15.8 \%)$. Los machos, los de razas puras y los adultos fueron significativamente más afectados por las patologías musculoesqueléticas.

Palabras clave: patologías músculo esqueléticas, caninos

\section{AbSTRACT}

A retrospective study of musculoskeletal pathologies in dogs was carried out at the German Kleintierklinik Veterinary Center in the city of San Pedro de la Paz, Chile, in the period September 2014 - June 2017. Of the total of 2000 clinical files analysed, 144 were related to patients with a problem in the musculoskeletal system. The musculoskeletal pathologies with the highest incidence were fractures $(40,27.8 \%)$, patellar dislocation $(31,21.5 \%)$, rupture of the cranial cruciate ligament $(25,17.4 \%)$ and hip dysplasia $(23$, $15.8 \%$ ). Males, purebreds, and adults were significantly more affected by musculoskeletal pathologies.

Key words: musculoeskeletal pathologies; dogs

\footnotetext{
${ }^{1}$ Escuela de Medicina Veterinaria, Sede Concepción, Universidad Santo Tomás, Chile

${ }^{2}$ E-mail: cfischer@santotomas.cl
}

Recibido: 3 de junio de 2019

Aceptado para publicación: 20 de marzo de 2019

Publicado: 11 de agosto de 2020 
INTRODUCCIÓN

En los últimos años se han reportado diversos estudios epidemiológicos relacionados con el sistema músculo esquelético, los cuales han investigado principalmente los factores de riesgo para enfermedades ortopédicas individuales (Beuing et al., 2000; Libardoni et al., 2016; O'Neill et al., 2016; Muir y De Rooster, 2017; Brice et al., 2018). Tales investigaciones son importantes para promover la comprensión de patologías específicas; sin embargo, no proporcionan información sobre la incidencia relativa y la importancia clínica de la enfermedad en comparación con otros trastornos músculo esqueléticos (Johnson et al., 1994). En la literatura actual existen pocas investigaciones relacionadas a la incidencia de las patologías ortopédicas, las que generalmente se asocian a especies específicas (Mendoza, 2006; Shearer, 2011; Purwanto et al., 2015; O’Neill et al., 2017).

Estudios relacionados a la incidencia de patologías, tanto a nivel local, nacional y mundial, son de importancia para realizar futuras investigaciones, dándole énfasis a investigaciones sobre las patologías más frecuentes, priorizando recursos de acuerdo con las patologías, como también dar relevancia de aprendizaje para los futuros médicos veterinarios. El objetivo del presente estudio fue proporcionar una visión general de la incidencia de lesiones a nivel del sistema músculo esquelético en caninos de un hospital veterinario, asociando las patologías con la edad, sexo y raza de los pacientes.

\section{Materiales y MéTOdos}

Los pacientes para el presente estudio fueron seleccionados de la base de datos del Centro Veterinario Alemán Kleintierklinik, ubicado en la Ciudad de San Pedro de La
Paz, Chile. Los pacientes caninos fueron atendidos entre el año 2014 y 2017. Los criterios de inclusión para la investigación fueron contar con la ficha clínica completa del paciente $\mathrm{y}$ tener un diagnóstico definitivo de una patología musculoesquelética. Los parámetros seleccionados a estudiar para la clasificación de los perros en grupos fueron: 1) Edad: cachorro (nacimiento y 12 meses); adulto (12 meses y 5 años de edad en razas grandes y 7 años en razas pequeñas); senil (mayor de 5 años para razas grandes y gigantes y mayor de 7 años para razas pequeñas) (Debraekeleer et al., 2000); 2) Sexo (macho, hembras); 3) Raza: puras, mestizos o sin información.

La información de los casos para todos los pacientes fue agrupada en tres conjuntos de datos: 1) huesos, 2) articulaciones y estructuras relacionadas, y 3 ) músculos y estructuras relacionadas, según el área del cuerpo (topografía). Primeramente se separaron las enfermedades basadas en topografía y tipo de tejido. Las enfermedades se agruparon en categorías basadas en etiología o trastornos patológicos. En los casos en que se especifique una reacción estructural o funcional de un tejido sin origen etiológico, el diagnóstico se clasificó en la categoría patológica, pero con causa no especificada. Los diagnósticos que no tuvieron una interpretación con una condición clínica conocida se agruparon en una categoría denominada «otras enfermedades» (Johnson et al., 1994). Finalmente, se registró el número total de pacientes con patologías músculos esqueléticas. La incidencia fue reportada en números absolutos y relativos.

El estudio fue de tipo retrospectivo y se elaboraron tablas de contingencia, presentándose los resultados en frecuencia relativa y absoluta, como en forma gráfica. Se utilizó el cálculo de frecuencia porcentual simple. Para determinar la asociación estadística entre las variables se utilizó la prueba de Chi cuadrado. 
Cuadro 1. Frecuencia de enfermedades músculo esqueléticas en caninos según diagnóstico de patologías específicas (Centro Veterinario Alemán Kleintierklinik, Chile)

\begin{tabular}{lcc}
\hline \multirow{2}{*}{ Frecuencia absoluta } & \multicolumn{2}{c}{$\begin{array}{c}\text { Pacientes } \\
\text { afectados }\end{array}$} \\
\cline { 2 - 3 } & $\mathrm{n}$ & $\%$ \\
\hline Fracturas & 40 & 27.8 \\
Luxación patelar & 31 & 21.5 \\
Ruptura ligamento & 25 & 17.4 \\
cruzado craneal & & \\
Displasia de cadera & 23 & 15.8 \\
Luxación coxofemoral & 10 & 7.0 \\
Panosteitis & 4 & 2.8 \\
Displasia de codo & 4 & 2.8 \\
Patologías musculares & 3 & 2.1 \\
Luxación de codo & 2 & 1.4 \\
Luxación sacroiliaca & 2 & 1.4 \\
\hline Total & 144 & 100 \\
\hline
\end{tabular}

\section{Resultados}

Se revisaron 2000 registros y se seleccionaron 144 que cumplieron con los criterios de inclusión escogidos. La patología más frecuente encontrada fue de fracturas (27.8\%), seguido de luxación patelar $(21.5 \%)$, ruptura del ligamento cruzado craneal (17.4\%) y displasia de cadera (15.8\%) (Cuadro 1).

Los resultados, según las variables edad, sexo y raza se describen en Cuadro 2. La población de pacientes fue predominantemente adulta, siendo el adulto el grupo etario más representado $(47.9 \%)$, seguido por perros de edad senil (40.3\%). Los machos fueron los más afectados $(61.8 \%)$, mientras que se observó una mayor frecuencia de canes afectados de razas puras (79.2\%) que de mestizos.

\section{Discusión}

Las patologías músculo esqueléticas de mayor presentación fueron principalmente las fracturas y luxaciones articulares; lo que concuerda con Bahamondes (2008), quienes identificaron nueve lesiones, dentro de las cuales las fracturas fueron los diagnósticos más frecuentes, seguido de las luxaciones en términos generales.

Se observó una mayor frecuencia de enfermedades músculo esqueléticas en machos con relación a las hembras. Chandia (2004) describe resultados semejantes ocurridos en el periodo 1996-1999, donde el $58.7 \%$ de 3121 registros clínicos de pacientes perros y gatos correspondieron a machos.

Cuadro 2. Frecuencia de enfermedades músculo esqueléticas en caninos según edad, sexo y raza (Centro Veterinario Alemán Kleintierklinik, Chile) $(\mathrm{n}=144)$

\begin{tabular}{lcc}
\hline \multirow{2}{*}{ Variable } & \multicolumn{2}{c}{ Pacientes } \\
\cline { 2 - 3 } & $\mathrm{n}$ & $\%$ \\
\hline Edad & & \\
\multicolumn{1}{c}{ Adultos } & 69 & 47.9 \\
$\quad$ Seniles & 58 & 40.3 \\
$\quad$ Cachorros & 15 & 10.4 \\
$\quad$ Sin & 2 & 1.4 \\
$\quad$ identificación & & \\
Sexo $\quad$ Macho & 89 & 61.8 \\
$\quad$ Hembra & 55 & 38.2 \\
Raza & & \\
$\quad$ Raza pura & 114 & 79.2 \\
$\quad$ Mestizos & 30 & 20.8 \\
\hline
\end{tabular}


Por otro lado, los resultados referentes a la edad difieren de los obtenidos por otros estudios. Así, Chandia (2004) encontró una mayor población de pacientes predominantemente joven, siendo el $87.7 \%$ menor a 8 años, especialmente el estrato etario de cachorros (0-11 meses); en tanto que Contreras (2008) encontró una mayor presentación en adultos $(49.4 \%)$ y seniles $(32.5 \%)$.

El estudio reveló una relación estadísticamente significativa entre la presencia de enfermedad ortopédica y animales de raza pura. En este sentido, González (2015) reportó 2.37 veces más casos en razas puras que en mestizos ( 9.42 vs. $3.97 \%$, respectivamente).

\section{Conclusiones}

- La mayor cantidad de pacientes asociadas a enfermedades músculo esqueléticas fue por fracturas seguidas por luxación patelar y displasia de cadera.

- Los perros machos, los adultos y los de raza pura fueron los más afectados por enfermedades musculoesqueléticas.

\section{Literatura Citada}

1. Bahamondes F. 2008. Estudio epidemiológico descriptivo de casos traumatológicos del servicio de cirugía de animales pequeños enero 1996-diciembre 2007. Tesis de Médico Veterinario. Santiago, Chile: Univ. de Chile. 60 p.

2. Beuing $\mathrm{R}$, Mues $\mathrm{CH}$, Tellhelm B, Erhardt G. 2000. Prevalence and inheritance of canine elbow dysplasia in German Rottweiler. J Anim Breed Genet 117: 375-383. Doi: 10.1046/j.14390388.2000.00267.x
3. Brice J, Lindvall E, Hoekzema N, Husak L. 2018. Dogs and orthopaedic injuries: is there a correlation with breed? J Orthop Trauma 32: e372-e375. doi: 10.1097/BOT.0000000000001235

4. Chandía AP. 2004. Estudio retrospectivo de registros clínicos caninos y felinos. Clínica de pequeños animales, Universidad de Chile, periodo 1996-1999. Tesis de Médico Veterinario. Santiago, Chile: Univ. de Chile. 104 p.

5. Contreras C. 2008. Estudio de imágenes radiográficas obtenidas de la casuística de perros del Hospital Veterinario de la Universidad Austral de Chile, Valdivia. Periodo 2000-2006. Tesis de Médico Veterinario. Valdivia, Chile. Univ. Austral de Chile. 44 p.

6. Debraekeleer J, Gross K, Zicker S. 2000. Perros normales. En: Hand M, Thatcher C, Remillard R, Roudebush P (eds). Nutrición clínica de pequeños animales. $4^{\circ}$ ed. Sta. Fe de Bogotá, Colombia: Mark Morris Institute. p 256-311.

7. González J. 2015. Relación de las patologías caninas más frecuentes que se presentan en la clínica de pequeños animales en la zona noroeste de la Comunidad de Madrid, con las variables edad, raza, sexo y tamaño. Tesis Doctoral. Madrid, España: Univ. Complutense de Madrid. 198 p.

8. Johnson JA, Austin C, Breur GJ. 1994. Incidence of canine appendicular musculoskeletal disorders in 16 veterinary teaching hospitals from 1980 through 1989. Vet Comp Orthopaed 7: 56-59.

9. Libardoni R, Serafini GM, Oliveira C, Schimites PI, Chaves RO, Feranti JP, Costa CA, et al. 2016. Appendicular fractures of traumatic etiology in dogs: 955 cases (2004-2013). Cienc Rural 46: 542-546. doi: 10.1590/0103-8478cr20150219 
10. Mendoza P. 2006. Prevalencia de displasia de cadera en caninos Ovejero Alemán y Labrador Retriever para adiestramiento. Tesis de Médico Veterinario. Santiago, Chile: Univ. de Chile. 44 p.

11. Muir P, De Rooster H. 2017. Advances in the canine cranial cruciate ligament. $2^{\text {nd }}$ ed. USA: Wiley-Blackwell. $109 \mathrm{p}$.

12. O'Neill DG, Meeson RL, Sheridan A, Church DB, Brodbelt DC. 2016. The epidemiology of patellar luxation in dogs attending primary-care veterinary practices in England. Canine Genetics Epidemiol 3: 4. doi: 10.1186/s40575-016-0034-0
13. O'Neill DG, Coulson NR, Church DB, Brodbelt DC. 2017. Demography and disorders of German shepherd dogs under primary veterinary care in the UK. Canine Genetics Epidemiol 4: 7. doi: 10.1186/s40575-017-0046-4

14. Purwanto DB, Susari NNW, Puja IK. 2015. Prevalence of musculoskeletal disorders in Kintamani dog. Vet Sci Med J 3: 65-68-

15. Shearer P. 2011. Epidemiology of orthopedic disease. Vet Focus 21: 24-25. 